journal club

\title{
Karotisendarteriektomie und Stenting zur Schlaganfallprävention ebenbürtig
}

Fragestellung: Sind Karotisendarteriektomie und Stenting in der Schlaganfallprävention bei Personen mit asymptomatischen Karotisstenosen gleich wirksam?

Hintergrund: Für Patienten mit symptomatischen Karotisstenosen ist der Nutzen der Operation und des Stentings zur Schlaganfallprävention gut belegt. In der Vergangenheit hatten die Studien Asymptomatic Carotid Atherosclerosis (ACAS) und Asymptomatic Carotid Surgery Trial (ACST) gezeigt, dass die Karotisoperation bei Personen mit über $60 \%$ igen Abgangsstenosen der Arteria carotis interna einen geringen Nutzen gegenüber der konservativen Therapie hat. Die CREST-Studie untersuchte bei einem Teil ihrer Patienten auch Personen mit asymptomatischen Karotisstenosen und fand keinen Unterschied in den Komplikationsraten und im Langzeitverlauf zwischen Operation und Stenting. Die Studie ACT-I (Asymptomatic Carotid Trial I) sollte die Frage der besseren Therapie bei Personen mit asymptomatischen Karotisstenosen in einer weiteren randomisierten Studie untersuchen.

Patienten und Methodik: Es handelte sich um eine randomisierte Studie bei maximal 79 Jahre alten Personen mit asymptomatischen Karotisstenosen,

Rosenfield K, Matsumura JS, Chaturvedi S et al. Randomized trial of stent versus surgery for asymptomatic carotid stenosis. N Engl J Med 2016; 374: $1011-20$ die innerhalb der letzten 180 Tage vor der Randomisierung keine zerebralen Durchblutungsstörungen hatten. Die Patienten wurden entweder unter Embolieprotektion gestentet oder operiert. Die Beobachtungszeit betrug fünf Jahre. Der primäre Endpunkt war die Kombination von Tod, Schlaganfall und Myokardinfarkt innerhalb von 30 Tagen nach der Intervention oder ein ipsilateraler Schlaganfall innerhalb eines Jahres. Die Randomisierung erfolgte im Verhältnis 3: 1 für Stenting und Operation. Wegen der langsamen Rekrutierung wurde die Studie nach dem Einschluss von 1.453 Patienten beendet.

Ergebnisse: Die Patienten waren im Mittel 68 Jahre alt, $60 \%$ waren Männer. Die Häufigkeit von vaskulären Begleiterkrankungen war in beiden Gruppen vergleichbar. Der mittlere Grad der Karotisstenose betrug $74 \%$.

Der kombinierte primäre Endpunkt trat bei 35 von $1.270 \mathrm{~Pa}-$ tienten der Stenting-Gruppe (3,3\%) und bei neun von 348 Patienten der Operationsgruppe (2,6\%) auf. Dieser Unterschied war statistisch nicht signifikant. Im Langzeitverlauf von fünf Jahren erlitten 2,2\% der Patienten in der Stenting- und 2,7\% in der Endarteriektomiegruppe einen ipsilateralen Schlaganfall. Auch dieser Unterschied war nicht signifikant. Die Rate der Patienten, die über fünf Jahre keinen Schlaganfall erlitten, betrug $93 \%$ in der Stenting- und $95 \%$ in der Endarteriektomiegruppe. Die Überlebensraten waren $87,1 \%$ und $89,4 \%$ und ebenfalls nicht signifikant unterschiedlich.

Schlussfolgerungen: Bei Patienten mit asymptomatischen hochgradigen Karotisstenosen ergaben sich bezüglich Komplikationsrate und Langzeitverlauf keine Unterschiede zwischen Operation und Stenting.

\section{- Kommentar von Hans-Christoph Diener, Essen}

\section{Es fehlt nach wie vor eine große randomisierte Studie}

Diese große Studie repliziert die Ergebnisse der Subgruppe aus der CREST-Studie bei Patienten mit asymptomatischen Karotisstenosen. Im Vergleich zu früheren Studien ist es bemerkenswert, wie gering in dieser aktuellen Untersuchung die Komplikationsrate beider Verfahren war. Daher wird bei Personen mit asymptomatischen Karotisstenosen die lokale Kompetenz bezüglich Gefäßchirurgie und Neuroradiologie die Entscheidung bestimmen, welcher Patient welcher Therapie unterzogen wird. Leider fehlt nach wie vor eine große randomisierte Studie, die interventionelle Verfahren mit einer optimalen medikamentösen Therapie verglichen hätte. Dies wäre notwendig, da sich in der Zwischenzeit nicht nur die Komplikationsraten der Eingriffe reduziert haben, sondern auch die medikamentösen Maßnahmen deutlich wirksamer sind. Allerdings wird eine solche Studie leider im Moment nicht durchgeführt.

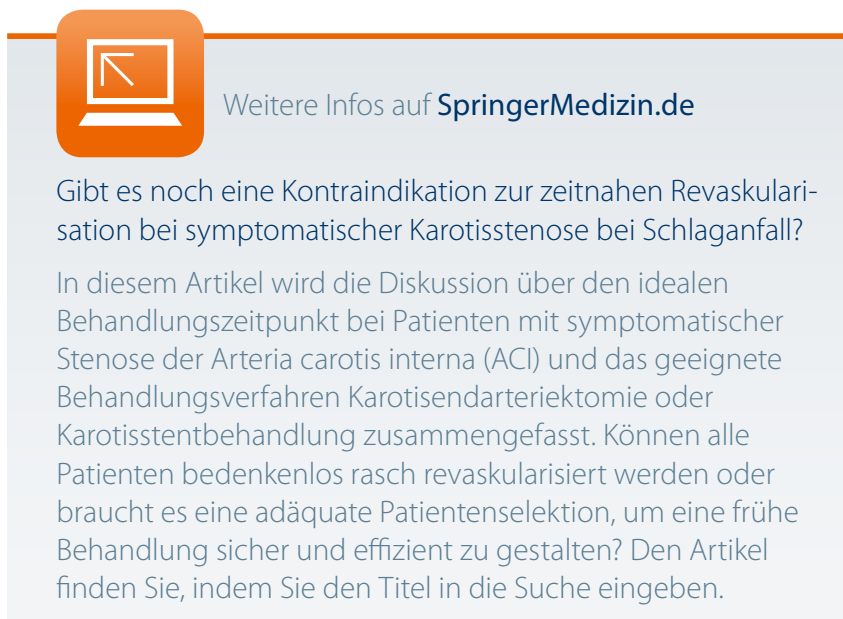

